was conducted among YMSM in two large cities of Myanmar in 2014 with the objective of determining the pattern of $S$ and D experienced by YMSM.

Methods A cross-sectional study was conducted by using quantitative and qualitative research methods. Respondent driven sampling was applied to recruit YMSM. Face-to-face interviews were done by using a structured questionnaire and in-depth interviews were also done with YMSM.

Results A total of 400 YMSM (200 each in Yangon and Monywa) included in the study. Their age ranged from 18-26 years with the median age of 22 . Over $42 \%$ self-identified as Apwint (feminine). Most YMSM (55.8\% and 69.6\%) reported having a regular job, but a higher percentage in Monywa reported an estimated monthly income of $>100,000$ Kyats compared to YMSM in Yangon $(\mathrm{p}<0.01)$. Around half $(48.2 \%$ and $51.5 \%)$ reported ever experiencing discrimination because of their same sex attraction. Among those who experienced discrimination, 60\% in Yangon and 53\% in Monywa reported experiencing discrimination exhibited as insults/verbal abuse and $(34 \%$ and $37 \%)$ reported discrimination exhibited as physical abuse or beating. Seventy percent of YMSM reported experiencing discrimination most often in public places. The largest percentages of YMSM who reported ever experiencing discrimination, were among Apwint (64\% and 50\%). "Exclusion from social occasion" and "beating" were indicated as the worst type of discrimination by the many YMSM in Yangon $(40 \%$ vs. $15 \%, \mathrm{p}<0.01)$ and Monywa $(47 \%$ vs. $27 \%$, $\mathrm{p}>0.05)$. Some YMSM stated that they are experiencing $S$ and D even among the family members and sometimes at health care settings resulting in delayed seeking care. They thought that people would not discriminate them if they become famous and could earn much money.

Conclusion S and D still existed towards YMSM especially in public places. Community awareness raising programs should be strengthened to reduce S and D towards YMSM.

\section{P4.73 ATTITUDES AND PRACTICES OF NURSES UPON THE DELIVERY OF HEALTH CARE TO COMMERCIAL SEX WORKERS (CSW) IN THE WESTERN PROVINCE, SRI LANKA}

${ }^{1} \mathrm{~N}$ Jayasinghe, ${ }^{2} \mathrm{~S}$ Tissera, ${ }^{3} \mathrm{~N}$ Silva,. ${ }^{1}$ Deputy Head - Nursing Faculty, International Institute of Health Sciences, Sri Lanka; ${ }^{23}$ rd year student, Nursing Faculty, International Institute of Health Sciences, Sri Lanka; ${ }^{3}$ Head of Academic Affairs, International Institute of Health Sciences, Sri Lanka

\subsection{6/sextrans-2017-053264.569}

Introduction The traditional socio-cultural norms of countries like Sri Lanka pose challenges to health care access to those engaged in tabooed and illegal professions like the commercial sex trade. CSWs accessing health services in similar countries have been proven to be of a low socio-economic background. Therefore it is important to prompt a behavioural change by placing strong policies in place to address the psychosocial issues of both the patient and the carer. Certain STI's like HIV, being immuno-compromising in nature, increase the susceptibility of many other illnesses, to which these CSWs should seek treatment promptly. In order to make policy level decisions to tailor the training of health care workers to improve acceptance of CSWs at their settings, it is first important to assess the current gaps in delivering holistic care to CSWs. The goal was to understand attitudes and perceptions of nurses (in multi-sectors) when attending to sexual and general health needs and delivering holistic health care to CSWs in the Western Province of Sri Lanka.

Methods A qualitative research which consisted of 3 focus group discussions were carried out on a total of 23 nurses from the government and private sectors where each group consisted of 7-8 participants with 2 moderators per group. Scenarios and questions were posed to evaluate the perceptions of nurses towards CSWs when delivering STI related and general health care.

Results Thematic analysis revealed three strong themes. They were, 'No difference in the level of care delivered', 'Social marginalisation based on assumptions' and 'Labelling and stereotyping of CSWs'. No difference was made in the level of care provided for CSWs compared to non-CSWs, or for STIs compared to other disease conditions. Though the level of care delivered is the same, the manner in which CSWs are 'made comfortable' in terms of 'holistic care' within the hospitals seemed less compared to other patients. Labelling and stereotyping were common practices, where the nurses did believe that their attitudes and behaviour could have a negative affect on health promotion and health seeking behaviour.

Conclusion It is better to invest on training nurses so that the CSWs are made to feel more accepted when accessing STI related and general health care needs.

\section{P4.74 ABSTRACT WITHDRAWN}

\section{P4.75 EXPLORING THE RELATIONSHIP BETWEEN SOCIAL FACTORS AND TREATMENT ADHERENCE AMONG CLINICALLY STABLE ART PATIENTS: STRUCTURAL EQUATION MODELLING TECHNIQUE}

${ }^{1}$ Ndumiso Tshuma, ${ }^{2}$ Peter Nyasulu. 'Community AIDS Response, Johannesburg, South African Republic; ${ }^{2}$ Wits University, Johannesburg, South African Republic

\subsection{6/sextrans-2017-053264.570}

Introduction Socio-cultural factors such as fear of disclosure of HIV status, lack of social support and fear of stigma from family are some of the aspects that prevent ART adherence amongst patients. Moreover, home stability and family support have been linked to better ART adherence. One study has revealed that males who had long-term housing, living with a partner and belonged to an HIV support group had better antiretroviral adherence. The study sought to establish the relationship between social factors and treatment adherence among adherence clubs members.

Methods A cross-sectional survey was conducted among 830 Adherence Club patients in Ekurhuleni, Gauteng. A closedended questionnaire was administered and the internal consistency of the scale was assessed by Cronbach's alpha coefficient. The proposed model was tested using structural equation modelling (AMOS software: ADC, Chicago, IL, USA).

Results Adherence clubs were found to improve treatment adherence among patients. Patients who were in adherence clubs are also receiving more support from their family members or the people they stay with. Perceptions and experiences of stigma and discrimination were found to be generally low among patients attending adherence clubs. These low levels of stigma and discrimination lead to an improvement in the levels of treatment adherence among the club members. 